\title{
Supporting Information for Self-assembly of fluorescent HIV capsid spheres for detection of capsid binders
}

Derrick Lau ${ }^{1}$, James C. Walsh ${ }^{1}$, Amir Mousapasandi ${ }^{1}$, Nicholas Ariotti², Vaibhav B. Shah ${ }^{1}$, Stuart Turville $^{3}$, David A. Jacques ${ }^{1}$ and Till Böcking ${ }^{1, *}$

1. EMBL Australia Node in Single Molecule Science and ARC Centre of Excellence in Advanced Molecular Imaging, School of Medical Sciences, UNSW Sydney, NSW 2052, Australia

2. Electron Microscope Unit, Mark Wainwright Analytical Center, UNSW Sydney, NSW 2052, Australia

3. The Kirby Institute, UNSW Sydney, NSW 2052, Australia

*Corresponding author: till.boecking@unsw.edu.au

\section{Supplementary Methods}

Stability of capsid spheres in buffer solutions with different salt concentrations. A. Unlabelled and labelled CA spheres were assembled from mixtures of CA N21C/A22C, N21C/A22C:K158CAF488 or N21C/A22C:K158C-AF647, diluted 5-fold with assembly buffer and centrifuged to collect particles (Beckman Coulter TLA120.1, $80000 \mathrm{rpm}, 20 \mathrm{~min}, 4^{\circ} \mathrm{C}$ ). After resuspension in a Tris buffer (50 mM, pH 8) containing $0,0.15,0.5$ or $1 \mathrm{M} \mathrm{NaCl}$, the CA spheres were incubated at room temperature for 2 hours and collected by centrifugation (Beckman Coulter TLA120.1, 80000 rpm, 20 $\min , 4^{\circ} \mathrm{C}$ ). The amount of CA in the pellet fractions was determined by SDS PAGE analysis with Coomassie staining followed by densitometry and expressed as a fraction of the amount of CA recovered for each $\mathrm{CA}$ composition after incubation with $1 \mathrm{M} \mathrm{NaCl}$.

Binding of CypA to CA spheres in the presence of cyclosporin A. CA spheres were immobilised on the surface inside microfluidic devices. A solution (30 $\mu \mathrm{L})$ containing AF488-CypA $(1 \mu \mathrm{M})$ and cyclosporin $A(10 \mu \mathrm{M})$ in imaging buffer was injected and incubated for 2 min. TIRF images were recorded in several fields of view (FOV) in both channels to measure the signals associated with CA spheres $(647 \mathrm{~nm})$ and co-localised CypA $(488 \mathrm{~nm})$. The solution was washed away with imaging buffer $(80 \mu \mathrm{L})$. Subsequently, the same biosensor experiment was repeated in the absence of cyclosporin A. Images were analysed using JIM to determine the median number of CypA bound per spheres (containing 3-8.5 fluorophores) in the presence and absence of cyclosporin $A$.

Structural integrity of CA spheres after incubation with CypA. Labelled CA spheres were assembled using $72 \mu \mathrm{M}$ CA N21C/A22C and $8 \mu \mathrm{M} \mathrm{K158C-AF647} \mathrm{in} 1 \mathrm{M} \mathrm{NaCl}$ in a Tris buffer $(50 \mathrm{mM}$, $\mathrm{pH}$ 8). CypA was added to $1.5 \mu \mathrm{L}$ of the CA sphere assembly solution, the mixtures were made up to a final volume of $10 \mu \mathrm{L}$, incubated for $\geq 5$ min and stained on a carbon formvar coated copper EM grid using uranyl acetate $(2 \% \mathrm{w} / \mathrm{v})$. Micrographs were collected using a FEI Tecnai G2 20 electron microscope.

TIRF image analysis. Dual colour TIRF images of CypA bound to CA spheres were analysed using the Generate_Multi_Channel_Traces script from JIM v4.3. Diffraction-limited spots were detected in the average intensity projection of the image stack using the following settings: cutof $f$, 0.3 or 0.5 ; channeltodetect, 2; mindistfromedge, 25; mincount, 10; maxcount, 1000000; mineccentricity, -0.1 ; maxeccentricity, 0.3 or 0.6 ; minlength, 0 ; maxDistFromLinear, 1000000; innerradius, 4.1; backgroundradius, 20. The number of fluorescent molecules in each detected spot was then calculated for both channels (CA and CypA) by dividing the fluorescence intensity of the spot by the corresponding calibration value obtained by single-molecule photobleaching. Equilibrium binding curves were obtained from TIRF images recorded at a range of CypA concentrations by plotting the median number of bound CypA molecules per sphere (defined as spots with 3-8.5 CA molecules) as a function of CypA concentration. The following 
equation was used for model fitting to obtain estimates for the dissociation constant and the maximum binding ratio, $\mathrm{B}(\mathrm{eq})=[\mathrm{CypA}] \times \mathrm{B}(\max ) /\left([\mathrm{CypA}]+\mathrm{K}_{\mathrm{D}}\right)$, where $\mathrm{B}(\mathrm{eq})$ is the number of bound CypA per spheres at equilibrium for a given CypA concentration, $[$ СурA] is the concentration of $C y p A, B(\max )$ is the number of bound CypA per sphere at saturation, and $K_{D}$ is the dissociation constant.

\section{Supplementary Figures}

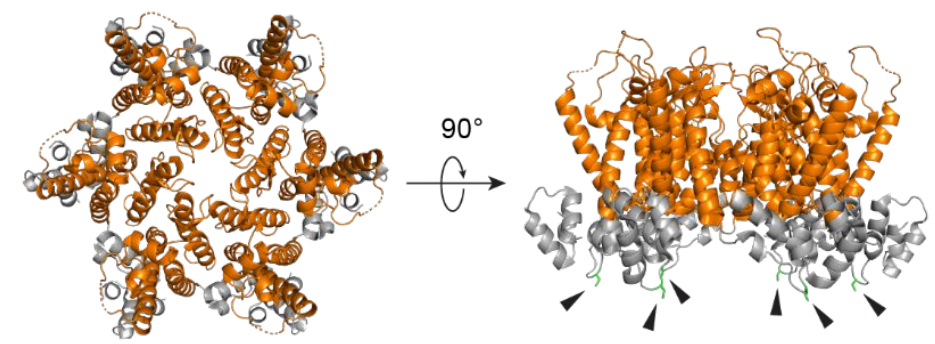

Figure S1. Engineering of the cysteine mutant on the CA for labelling. Crystal structure of the CA hexamer with the NTD and CTD coloured in orange and gray, respectively. Lysine residues at position 158 in the CTD of CA are away from the assembly interfaces and were mutated to cysteines for labelling (black arrows). The figure was generated from PDB $5 \mathrm{HGN}^{1}$ by replacing lysine at 158 with cysteine using Pymol.
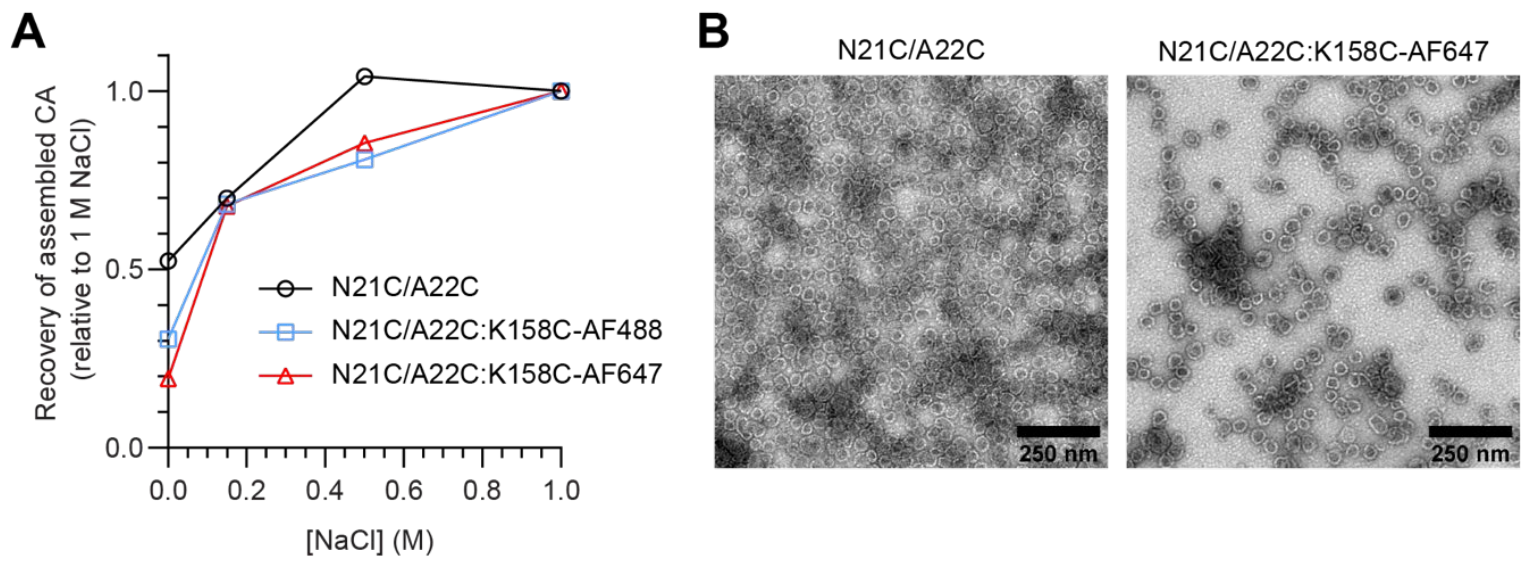

Figure S2. Stability of capsid spheres in buffer solutions with different salt concentrations. A. Recovery of capsid spheres after incubation in buffer solutions containing $0,0.15,0.5$ or $1 \mathrm{M} \mathrm{NaCl}$ for 2 hours at room temperature. B. Negative staining EM images of spheres collected after incubation with $0.15 \mathrm{M} \mathrm{NaCl}$ for 2 hours at room temperature. 

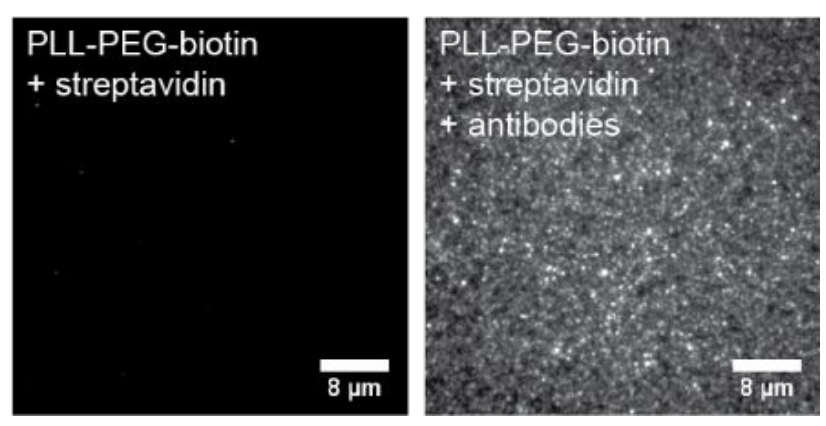

Figure S3. Specific adsorption of CA spheres onto modified surfaces. Total internal reflection fluorescence image of a surface after injection of CA N21C/A22C:CA K158C-AF568 spheres on surfaces modified without (left) and with (right) antibodies directed against the N-terminus of CA.

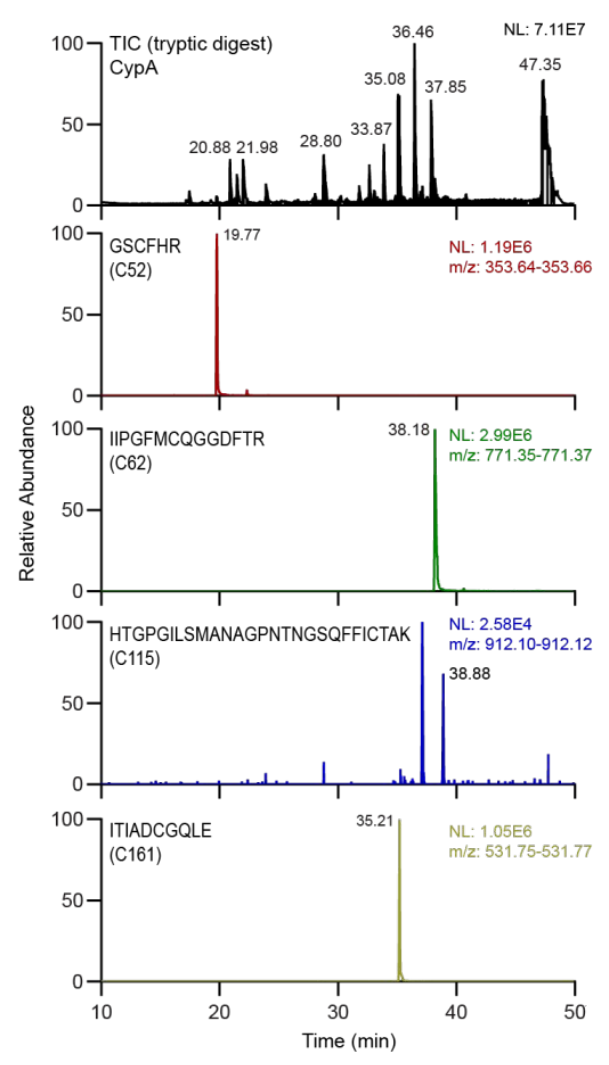

Figure S4. Total ion chromatogram of trypsinized CypA and extracted ion chromatograms for the peptides containing C52 $\left(\mathrm{m} / \mathrm{z}=353.66,(\mathrm{M}+2 \mathrm{H})^{2+}\right), \mathrm{C} 62\left(\mathrm{~m} / \mathrm{z}=771.37,(\mathrm{M}+2 \mathrm{H})^{2+}\right), \mathrm{C} 115(\mathrm{~m} / \mathrm{z}=$ 912.11, $\left.(\mathrm{M}+3 \mathrm{H})^{3+}\right)$ and $\mathrm{C} 161\left(\mathrm{~m} / \mathrm{z}=531.76,(\mathrm{M}+2 \mathrm{H})^{2+}\right)$ from LC-MS/MS. Peptides were detected on the basis of the expected mass-to-charge $(\mathrm{m} / \mathrm{z})$ ratio and identified using multiple $b$ - and $y$-ions in the secondary mass spectra. LC-MS/MS analysis of trypsinized AF568-CypA and AF647-CypA showed the presence of peptides containing $\mathrm{C} 62$, C115 and $\mathrm{C} 161$ but the peptide containing $\mathrm{C} 52$ was below the detection limit. Instead modified peptides with the added mass of AF568 or AF647 (Table S1) were detectable (see Figure 4C). Dye-labelled peptides containing C62, C115 or C161 were not detectable. Taken together these data suggest that the dye was exclusively incorporated at C52. 
Table S1. Monoisotopic mass of molecules for mass spectrometry analysis

\begin{tabular}{|l|l|l|}
\hline Molecule & Peptide sequence or molecular formula & Monoisotopic mass (M) \\
\hline Peptide with Cys52 & GSCFHR & 706.3089 \\
\hline Peptide with Cys62 & IIPGFMCQGGDFTR & 1541.7239 \\
\hline Peptide with Cys115 & HTGPGILSMANAGPNTNGSQFFICTAK & 2734.3075 \\
\hline Peptide with Cys161 & ITIADCGQLE & 1062.5136 \\
\hline AF568 & $\mathrm{C}_{42} \mathrm{H}_{42} \mathrm{~N}_{4} \mathrm{O}_{12} \mathrm{~S}_{2}$ & 858.2241 \\
\hline AF647 & $\mathrm{C}_{41} \mathrm{H}_{48} \mathrm{~N}_{4} \mathrm{O}_{16} \mathrm{~S}_{4}$ & 980.23 \\
\hline
\end{tabular}
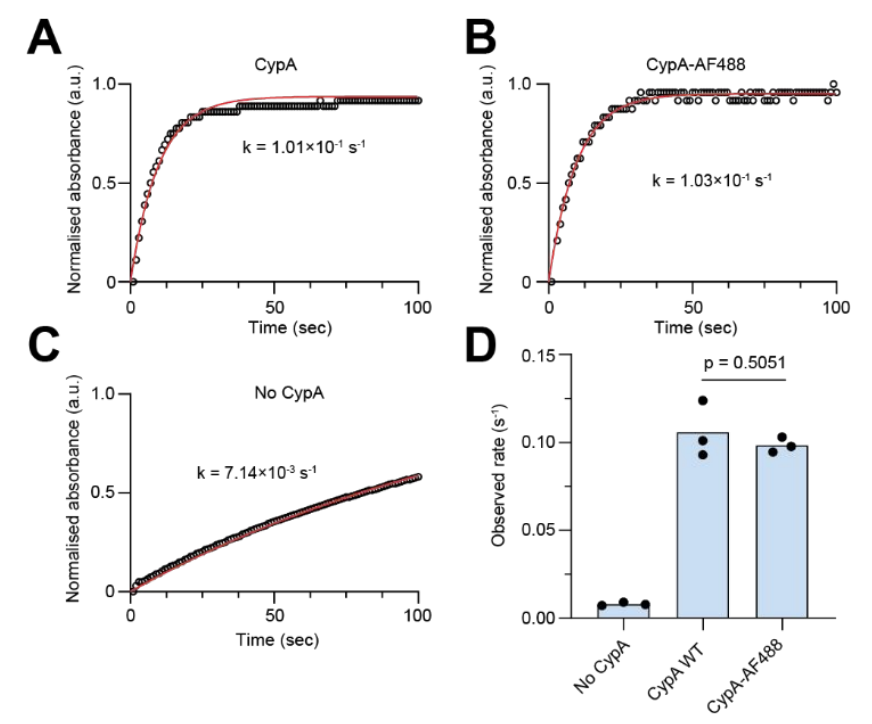

Figure S5. CypA labelled at C52 remains catalytically active. A-C. Reaction kinetics of cis-trans peptidyl-prolyl isomerisation measured in a chymotrypsin-coupled colorimetric assay (absorbance at $390 \mathrm{~nm}$ ) in the presence of $0.5 \mu \mathrm{M}$ CypA (A), $0.5 \mu \mathrm{M}$ AF488-CypA (B) and without CypA (C). D. Observed isomerisation rates, each symbol represents an independent assay $(N=3)$; unpaired t-tests with Welch's correction. 

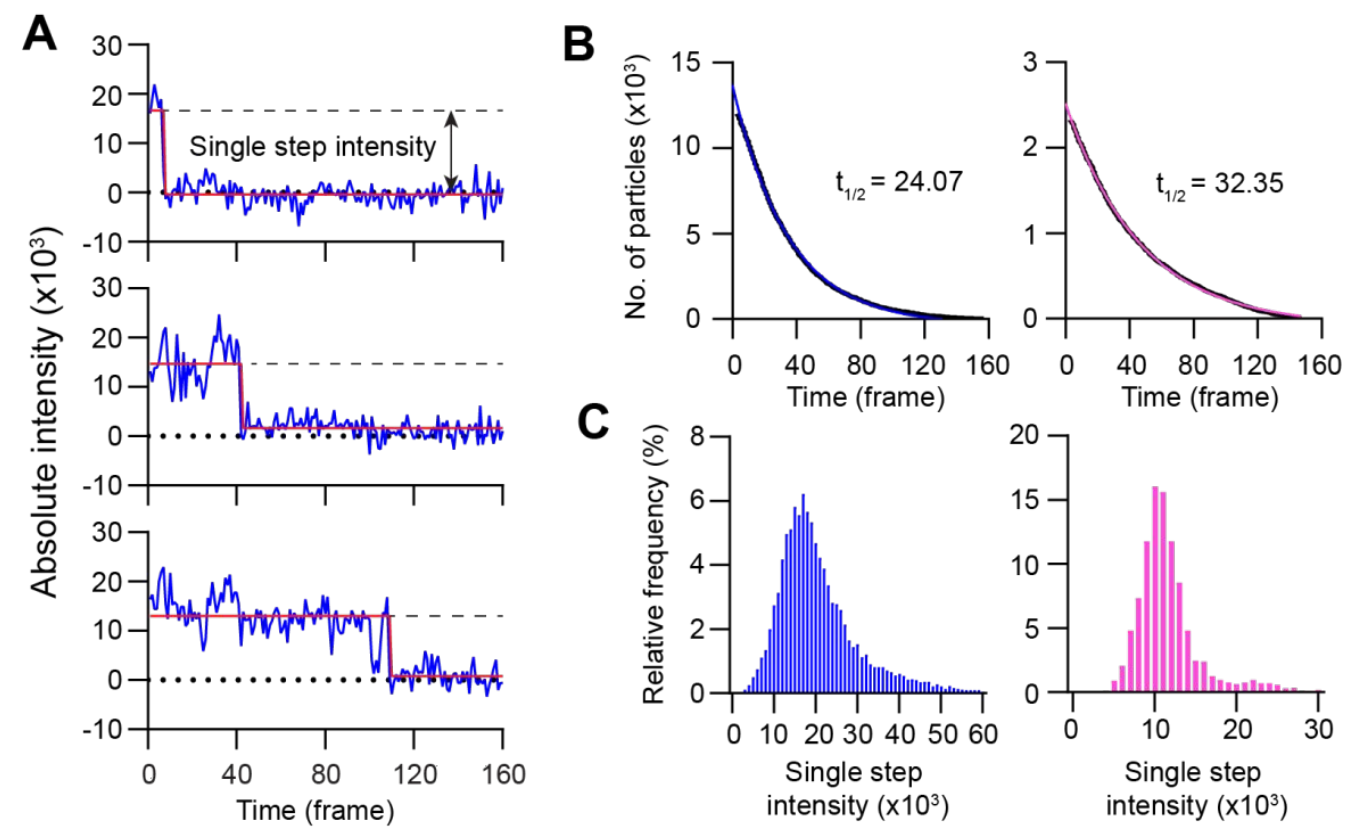

Figure S6. Photobleaching analysis of labelled CypA and CA K158C. A. Representative single molecule photobleaching traces of AF488-CypA. Background-corrected intensity traces (blue) were fitted with a step detection algorithm (red). B. Photobleaching kinetics of AF488-CypA molecules (blue, $500 \mathrm{~ms} / \mathrm{frame}$ ) and CA K158C-AF647 (magenta, $300 \mathrm{~ms} /$ frame). C. Histograms of single step intensities obtained from photobleaching analysis of AF488-CypA (blue) and CA K158C-AF647 (magenta). The histograms were fitted with a Gaussian distribution to extract the mean single molecule intensity; 17126 for AF488-CypA $(N=11982)$ and 10538 for CA K158C-AF647 $(N=2325)$.

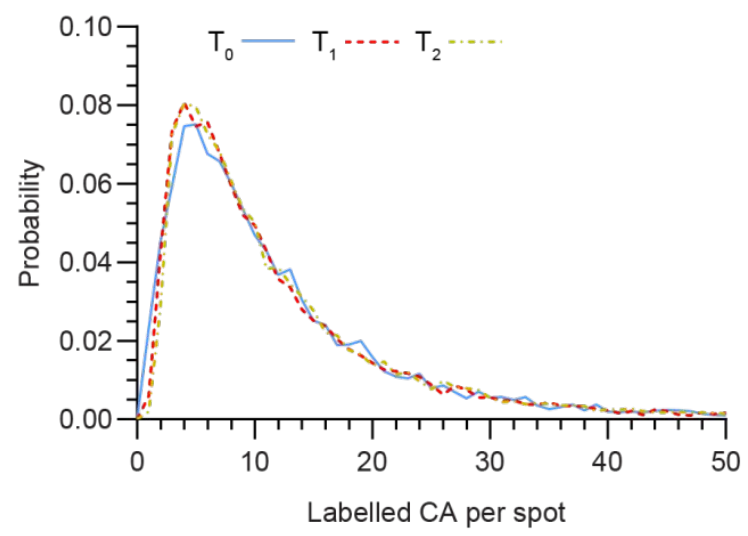

Figure S7. Labelled CA K158C remains stably associated with the spherical CA lattice. Distributions of the number of labelled CA molecules per spot in TIRF images collected immediately after deposition onto the coverslip surface $\left(T_{0}\right.$, blue, $\left.N=10028\right)$, after incubation for 1 hour $\left(T_{1}\right.$, red, $N$ $=14930)$ and 2 hours $\left(T_{2}\right.$, yellow, $\left.N=11414\right)$ in imaging buffer. Data recorded in two different microfluidic devices were combined. The distributions showed no shift to lower intensities indicating that the labelled CA K158C subunits were stably associated with the spherical lattice. 


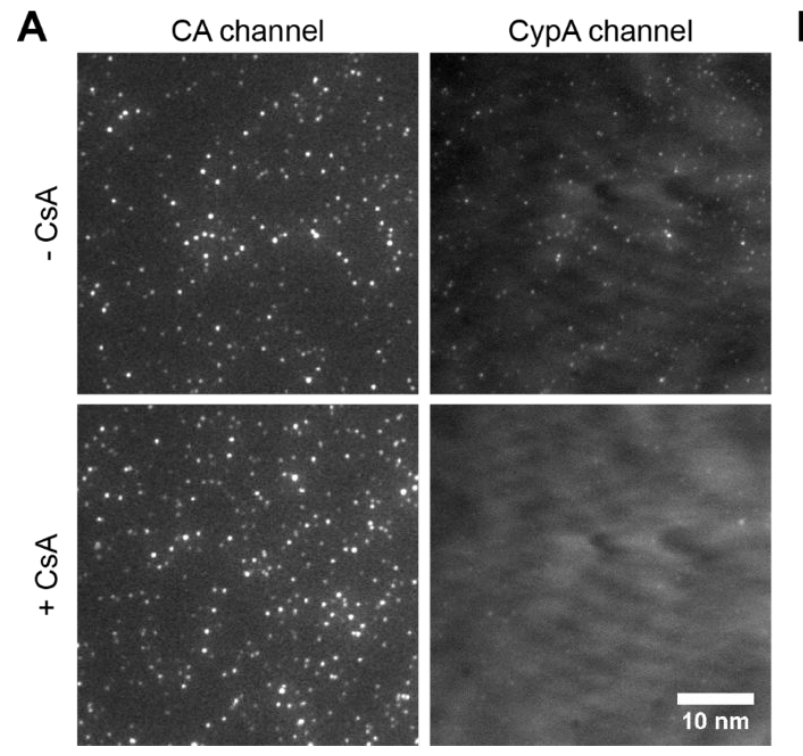

B

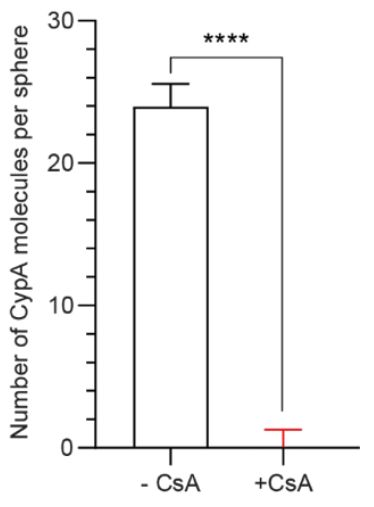

Figure S8. Cyclosporin A inhibits CypA binding to capsid spheres. A. Representative TIRF images of the CypA and CA channels after injection of solution containing AF488-CypA $(1 \mu \mathrm{M})$ in the absence and presence of the competitive inhibitor cyclosporin A (CsA, $10 \mu \mathrm{M})$. B. Bar graphs of AF488-CypA bound per spheres in the absence $(N=16$ fields of view) and presence $(N=18$ fields of view) of $10 \mu \mathrm{M}$ CsA. Data were collected from three microfluidic flow cells. Errors bars represent the standard deviation, statistical comparison using a two-tailed Welch's t-test $(p<0.0001)$.

- СурA

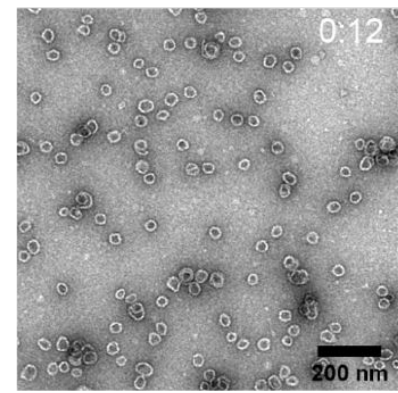

$25 \mu \mathrm{M}$ CурА

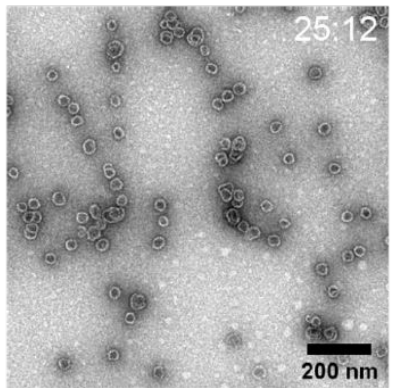

$50 \mu \mathrm{M}$ СурА

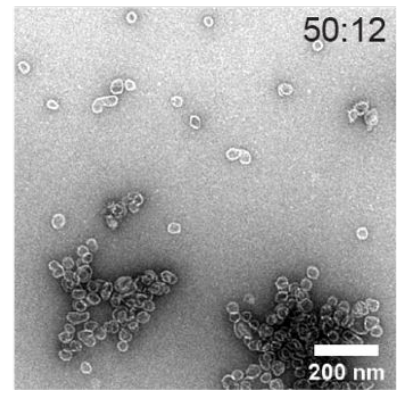

Figure S9. Structural integrity of CA spheres after incubation with CypA. Representative negative staining electron micrographs of CA N21C/A22C:CA K158C-AF647 spheres incubated without CypA (left), with $25 \mu \mathrm{M}$ CypA (middle) or $50 \mu \mathrm{M}$ CypA (right). The molar ratio of CypA:CA is indicated in the top right corner. Three independent incubations were performed with identical results. 
A
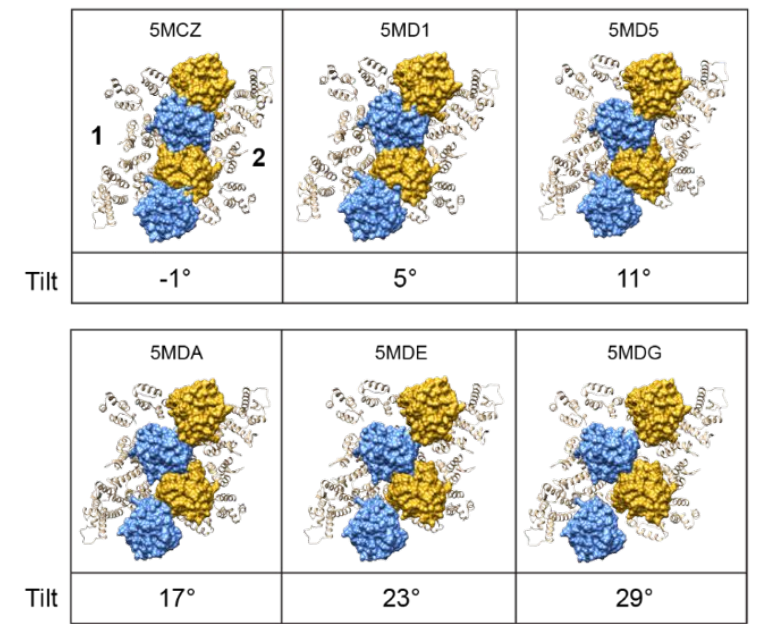

B

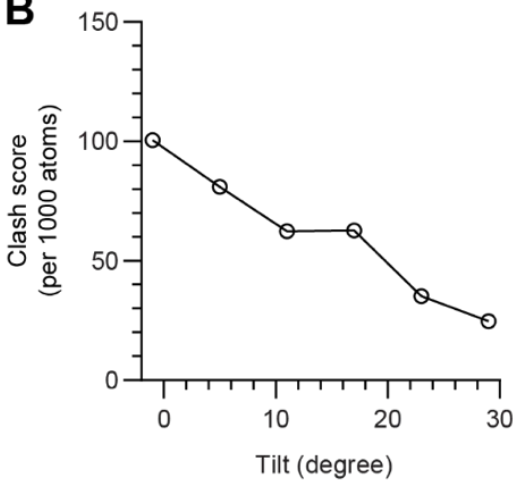

Figure S10. The steric clashes between bound CурA molecules decrease with increasing tilt between adjacent CA hexamers. A. Models showing CypA molecules bound to CypA loops at the inter-hexamer junction between hexamer 1 (blue) and hexamer 2 (gold) at different tilt. Models were generated by superimposing the crystal structure of the N-terminal domain of CA in complex with CypA (PDB 1M9C) $)^{2}$ onto cryo-EM models of the inter-hexamer junction with varying tilt observed in native virions (PDB 5MCZ, 5MD1, 5MD5, 5MDA, 5MDE, 5MDG) ${ }^{3}$. Note that the highly curved regions at the ends of the capsid can exhibit higher tilt angles (up to $\left.\sim 40^{\circ}\right)^{4}$ between adjacent hexamers. B. Plot showing the clash score between the CypA molecules as a function of tilt for the models shown in A. The clash score was calculated using Molprobity ${ }^{5}$ (http://molprobity.biochem.duke.edu/).

\section{Supporting Information References}

1. Jacques, D. A.; McEwan, W. A.; Hilditch, L.; Price, A. J.; Towers, G. J.; James, L. C., Hiv-1 Uses Dynamic Capsid Pores to Import Nucleotides and Fuel Encapsidated DNA Synthesis. Nature 2016, 536, 349.

2. Howard, B. R.; Vajdos, F. F.; Li, S.; Sundquist, W. I.; Hill, C. P., Structural Insights into the Catalytic Mechanism of Cyclophilin A. Nat Struct Biol 2003, 10 (6), 475-481.

3. Mattei, S.; Glass, B.; Hagen, W. J.; Kräusslich, H. G.; Briggs, J. A., The Structure and Flexibility of Conical Hiv-1 Capsids Determined within Intact Virions. Science 2016, 354 (6318), 14341437.

4. Zhang, Z.; He, M.; Bai, S.; Zhang, F.; Jiang, J.; Zheng, Q.; Gao, S.; Yan, X.; Li, S.; Gu, Y.; Xia, N., T = 4 Icosahedral Hiv-1 Capsid as an Immunogenic Vector for Hiv-1 V3 Loop Epitope Display. Viruses 2018, 10 (12).

5. Williams, C. J.; Headd, J. J.; Moriarty, N. W.; Prisant, M. G.; Videau, L. L.; Deis, L. N.; Verma, V.; Keedy, D. A.; Hintze, B. J.; Chen, V. B.; Jain, S.; Lewis, S. M.; Arendall, W. B., 3rd; Snoeyink, J.; Adams, P. D.; Lovell, S. C.; Richardson, J. S.; Richardson, D. C., Molprobity: More and Better Reference Data for Improved All-Atom Structure Validation. Protein Sci 2018, 27 (1), 293-315. 